\title{
Avaliação de indutores de resistência no controle de Dactylopius opuntiae em genótipos de Opuntia
} spp.

\section{Raquel Maria da Silva ${ }^{1}$, Edcleyton José de Lima ${ }^{2}$, Djalma Cordeiro dos Santos ${ }^{3}$, Cesar Auguste Badji ${ }^{4}$, João Tiago Correia Oliveira ${ }^{5}$ e Keila Aparecida Moreira ${ }^{4}$}

\footnotetext{
${ }^{1}$ Universidade Federal do Agreste de Pernambuco. Programa de Pós-Graduação em Produção Agrícola. Av. Bom Pastor, S/№. Boa Vista. Garanhuns-PE, Brasil (CEP 55292-270). E-mail: raquel.maria18@hormail.com.

${ }^{2}$ Universidade Federal do Agreste de Pernambuco. Curso de Graduação em Engenharia Agronômica. Av. Bom Pastor, S/№. Boa Vista. Garanhuns-PE, Brasil (CEP 55292-270).

${ }^{3}$ Instituto Agronômico de Pernambuco. Estação Experimental de Arcoverde. BR 232, km 253. São Miguel Arcoverde-PE, Brasil (CEP 56500-000).

${ }^{4}$ Universidade Federal do Agreste de Pernambuco. Av. Bom Pastor, S/№. Boa Vista. Garanhuns-PE, Brasil (CEP 55292-270).

5Universidade Federal do Sul e Sudeste do Pará. Instituto de Estudos do Trópico Úmido. Rua Alberto Santos Dumont, S/№. Jardim Universitário. Xinguara-PA, Brasil (CEP 68557-335).
}

Resumo. Produtos de origem biótica e abiótica estão sendo investigados para utilização no manejo de pragas. A busca por formas eficientes de controle da cochonilha-do-carmim Dactylopius opuntiae Cockerell que infestam a palma forrageira Opuntia spp. a qual é responsável pela destruição de palmais em todo Nordeste sendo considerada uma praga-chave, que afeta fortemente a pecuária no semiárido, faz-se cada vez mais necessárias. 0 objetivo do presente estudo foi avaliar a aplicação de indutores de resistência bióticos e abióticos no controle da infestação da cochonilha do carmim $D$. opuntiae. 0 experimento foi conduzido em casa de vegetação em condições controladas na Universidade Federal do Agreste de Pernambuco. Foi utilizado o delineamento inteiramente casualizado, distribuído em esquema fatorial ( $3 \times 8 \times 4)$, sendo três genótipos de palma (F8, IPA 200008, Opuntia atropes, Rose; IPA clone 20, IPA 100003 O. ficus indica e IPA 200016, O. stricta (Haw.) Haw), oito tratamentos, Trichoderma viride URM 6824, Trichoderma viride URM 6823, Aureobasidium pullulans URM 6874, ácido salicílico (AS), quitosana, ácido amino butírico (BABA), água destilada com o inseto (testemunha positiva), água destilada sem o inseto (testemunha negativa), com quatro períodos de avaliação e quatro repetições. Foram utilizadas 50 colônias por planta na qual foram fixadas com auxílio de um cordão evitando assim

Recebido

$23 / 12 / 2020$

Aceito

$25 / 04 / 2021$

Publicado

$30 / 04 / 2021$

Acesso aberto

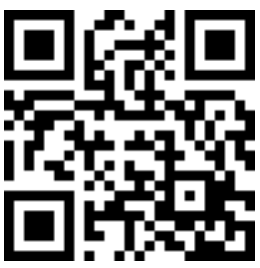

ISSN 2359-1412/RBGAS-2020-0181/2021/8/18/34/533

Rev. Bras. Gest. Amb. Sustent.

http://revista.ecogestaobrasil.net 
ferimentos aos tecidos. Os resultados foram submetidos à análise de variância, sendo as médias comparadas pelo teste de Scott-Knott ao nível de $5 \%$ de probabilidade $(\mathrm{p}<0,05)$, empregando o software estatístico Sisvar. No quarto período de avaliação o genótipo 0 . atropes tratadas com $T$. viride URM 6824, quitosana e BABA apresentaram melhores quantitativos de colônias, para O. ficus indica foram as com T. viride URM 6824, quitosana e AS, com 706, 655,75 e 719, respectivamente, enquanto a média de colônia nas 0 . stricta foi de 58,64 ao final do experimento, tais resultados evidenciam as potencialidades do uso de indutores no manejo da praga.

Palavras-chave: Cochonilha-do-carmim; Infestação artificial; Palma forrageira.

Abstract. Evaluation of resistance inducers in the control of Dactylopius opuntiae in genotypes of Opuntia spp. Products of biotic and abiotic origin are being investigated for use in pest management. The search for efficient ways to control the carmine cochineal Dactylopius opuntiae Cockerell that infest the forage palm Opuntia spp. is responsible for the destruction of palm trees throughout the Northeast Brazil and is considered a key pest that strongly affects livestock in the semiarid region become increasingly necessary. The aim of the present study was to evaluate the application of biotic and abiotic resistance inducers to control infestation of carmine cochineal D. opuntiae. The experiment was carried out in a greenhouse under controlled conditions at the Federal University of Agreste in Pernambuco. A completely randomized design was used, distributed in a factorial scheme ( $3 \times 8 \times 4)$ with three palm genotypes (F8, IPA 200008, Opuntia atropes, Rose; IPA clone 20, IPA 100003 O. ficus indica and IPA 200016, O. stricta (Haw.) Haw), eight treatments: Trichoderma viride URM 6824, Trichoderma viride URM 6823, Aureobasidium pullulans URM 6874, salicylic acid (AS), chitosan, amino butyric acid (BABA), distilled water with the insect (positive control), distilled water without the insect (negative control), with four evaluation periods and four repetitions. Fifty colonies were used per plant in which they were fixed with the aid of a cord, thus avoiding tissue injuries. The results were submitted to analysis of variance, and the means were compared using the Scott-Knott test at the level of $5 \%$ probability $(p<0.05)$, using the Sisvar statistical software. In the fourth evaluation period, the $O$. atropes treated with $T$. viride URM 6824, chitosan and BABA showed better colony numbers, for the 0 . ficus indica they were with $T$. viride URM 6824, chitosan and AS, with 706; 655.75 and 719 respectively while the average colony in 0 . stricta was 58.64 the end of the experiment. such results show the potential of using inductors in pest management.

Keywords: Carmine mealybug; Artificial infestation; Forage palm.
ORCID

(1) 0000-0002-5503-2072

Raquel Maria da Silva

(ㄷ) 0000-0002-3635-1369

Edcleyton José de

Lima

(D) 0000-0002-1194-300X

Djalma Cordeiro dos

Santos

D 0000-0002-7715-9285

Cesar Auguste Badji

(อ) 0000-0001-7469-5106

João Tiago Correia

Oliveira

(D) 0000-0002-7715-9285

Keila Aparecida

Moreira 


\section{Introdução}

A palma forrageira é uma dicotiledônea perene que pertence à Família Cactaceae e tem como centro de origem o México, atualmente encontra-se dispersa em todos os continentes no globo terrestre, exceto nas regiões polares. No Brasil o vegetal é cultivado predominantemente para utilização como recurso forrageiro, sendo considerado uma das principais fontes nutricionais para os ruminantes, pois apresenta uma elevada produção de biomassa por unidade de área, impactando diretamente na sustentabilidade da pecuária leiteira da região Nordeste do país (Marques et al., 2017).

Estima-se que há cerca de 600.000 ha sendo cultivados no país, porém os aspectos fitossanitários, principalmente as pragas são consideradas um dos principais entraves na exploração agrícola, destacando-se a cochonilha do carmim (Dactylopius opuntiae Cockerell), constituindo assim uma praga chave da cultura no Brasil (Lira, 2017; Silva et al., 2019). Palafox-Luna et al. (2018) afirmaram que uma infestação maior que $75 \%$ acarretam em morte da planta. Além de danos severos oriundos do ataque, há também sinergismo com agentes fitopatogênicos como os fungos e as bactérias.

$\mathrm{Na}$ agricultura moderna há possibilidade de realizar a indução da resistência, diversos estudos já comprovaram eficiência em diversas plantas. A indução ocorre através da aplicação de elicitores que podem ser de origem biótica (microrganismos viáveis ou inativados) e abiótica (Pascholati et al., 2019). Podendo ser aplicados na parte área e no solo, próximo ao sistema radicular de absorção (Melo et al., 2016). São chamados também fortalecedores de plantas, podendo proteger contra o ataque de pragas e patógenos, esses agentes externos aumentam o rendimento das culturas. A maioria atua modificando as vias de sinalização de defesa vegetal, porém pouco se sabe sobre como ocorre as reações em cadeias na ativação da sinalização (Wang et al., 2020).

Diversos micro-organismos possuem a capacidade de eliciar o sistema de defesa vegetal, dentre eles os mais estudados pertencem às espécies de Trichoderma (Pascholati et al., 2019). A indução de defesas nas plantas por espécies de Trichoderma ocorre de forma sistêmica, frente ao ataque seja de patógenos ou insetos. Podendo ocorrer em condições edafoclimáticas adversas, não havendo a necessidade do estabelecimento de contato direto com o invasor ou um estresse prévio. A. Pullulans possuem ampla ação já descobertas em experimentos com plantas, dentre elas estão a produção de enzimas hidrolíticas (Ferraz et al., 2016), produção de micotoxinas (Pfliegler et al., 2015) e produção de alguns compostos voláteis (Bozoudi e Tsaltas, 2018).

0 ácido salicílico é um agente abiótico que tem relação com a eliminação de espécies reativas de oxigênio e também age equilibrando sua produção, estando ligada no melhoramento da fotossíntese e na atividade enzimática (Batista et al., 2019). A quitosana constitui indução de resistência em plantas, principalmente por produzir compostos e enzimas que atuam contra patógenos (Gorri et al., 2017). 0 ácido amino butírico (BABA) tem efeitos anti-patogênicos registrados em fungos, bactérias e insetos e contribui significativamente para o aumento da resistência induzida em plantas (Delgado-Ormas et al., 2020).

Diante do exposto, o objetivo deste estudo foi avaliar a utilização de indutores bióticos (Trichoderma spp e Aureobasidium pullulans) e abióticos (quitosana, ácido salicílico e o ácido amino butírico) no controle da infestação da cochonilha-do-carmim (D. opuntiae) em três genótipos de palma forrageira do gênero Opuntia.

\section{Material e métodos}

O experimento foi conduzido sob condições controladas em casa de vegetação da Universidade Federal do Agreste de Pernambuco (UFAPE), no município de Garanhuns situado na mesorregião do Agreste Meridional do estado de Pernambuco no Brasil, sob as 
coordenadas geográficas de referência $08^{\circ} 53^{\prime} 25^{\prime \prime}$ de latitude sul e $36^{\circ} 29^{\prime} 34^{\prime \prime}$ de longitude oeste, com altitude média de $896 \mathrm{~m}$ (Canuto et al., 2019).

0 clima da região é classificado como Mesotérmico Tropical de Altitude (Cs'a) de acordo com classificação de Köppen-Geiger, apresenta temperatura média de $20,4^{\circ} \mathrm{C}$ com pluviosidade média anual de $873 \mathrm{~mm}$, caracterizado por verões quentes e secos, e invernos amenos e úmidos, a umidade relativa do ar varia entre $73 \%$ e $83 \%$ (Clima.Date.Org, 2020). Durante o período experimental de 13 de agosto de 2019 a 26 de março de 2020, com duração de 226 dias. Segundo o INMET (2020) a temperatura teve uma média de $23,11^{\circ} \mathrm{C}$ e umidade de $80,54 \%$.

Os fungos utilizados foram Trichoderma viride URM 6824, Trichoderma viride URM 6823 e Aureobasidium pullulans URM 6874, oriundos da coleção de Culturas Micoteca URM, do Departamento de Micologia, do Centro de Biociências, da Universidade Federal de Pernambuco (UFPE), os isolados de Cereus jamacaru DC. (Mandacaru, Cactaceae) no Município de Santa Teresinha no Estado da Paraíba. Os microrganismos foram cultivados para crescimento em frascos do tipo Erlenmeyer de $250 \mathrm{~mL}$, contendo $50 \mathrm{~mL}$ de meio de cultura sólido Batata-Dextrose-Ágar (BDA). O crescimento ocorreu em uma estufa incubadora tipo B.O.D. por 15 dias no Laboratório de Microbiologia, Tecnologia Enzimática e Bioprodutos (LMTEB), da UFAPE.

O solo no qual foram cultivadas as plantas foi coletado na UFAPE em profundidade entre 0-20 cm, onde foi homogeneizada e acondicionada em saco plástico, e posteriormente encaminhada ao laboratório para realizações das análises químicas e físicas (Tabela 1). Para as análises de solo aplicou-se a metodologia de Teixeira et al. (2017).

No estudo foram utilizadas três cultivares F8, IPA 200008, Opuntia atropes, Rose; IPA clone 20 (Tolerante), IPA 100003 O. ficus indica (Susceptível) e IPA 200016, O. stricta (Haw.) Haw (resistente). Os materiais propagativos (cladódios) foram obtidos do banco de germoplasma in vivo do Instituto Agronômico de Pernambuco (IPA), localizado no Município de Arcoverde-PE. Após a colheita, os cladódios foram higienizados com solução de hipoclorito de sódio a $2 \%$ e detergente neutro a $20 \%$, em seguida foram armazenados durante 19 dias em local seco, e ao abrigo da radiação solar direta, para a cicatrização do corte, com o objetivo de evitar contaminações e apodrecimento.

As plantas foram cultivadas em vasos com capacidade de $20 \mathrm{~L}$ contendo solo e irrigadas de acordo com a capacidade de campo, aos 60 dias de estabelecimento da planta nos vasos, foi realizado um desbaste para uniformização, deixando apenas os cladódios primários em cada planta, assim após 136 dias do plantio, os cladódios foram pulverizados até o ponto de escorrimento com soluções dos indutores de resistência, também foi aplicado via solo $40 \mathrm{~mL}$ de cada indutor, as aplicações aconteceram simultaneamente no período noturno (Figura 1).

Os indutores ácido salicílico $(2 \mathrm{mM})$, quitosana $\left(4 \mathrm{mg} \mathrm{mL}^{-1}\right)$ e DL- $\beta$-amino-nbutírico (BABA) (3 mM), considerados indutores abióticos, e duas espécies de Trichoderma viride (URM 6824 e URM 6823) e uma de Aureobasidium pullulans (URM 6874) ambos na concentração de esporos $10^{6}$ como indutores bióticos e duas testemunhas, $\mathrm{H}_{2} \mathrm{O}$ destilada e outra $\mathrm{H}_{2} \mathrm{O}+$ cochonilha do carmim, com quatro repetições.

Dez dias após a indução, as plantas foram infestadas artificialmente com exemplares de $D$. opuntiae, exceto as testemunhas negativas. Os insetos foram oriundos do IPA, Estação Arcoverde, e mantidos em criação no Laboratório de Entomologia Aplicada, do Centro Laboratorial de Apoio à Pesquisa da UFAPE. A infestação ocorreu por meio de fixação de fragmentos de cladódios com dimensões de $8 \mathrm{~cm} \times 5 \mathrm{~cm} \pm 2 \mathrm{~cm}$ contendo 50 colônias do inseto em estágio de postura (Figura 2) as colônias foram fixadas nas plantas com cordões. 


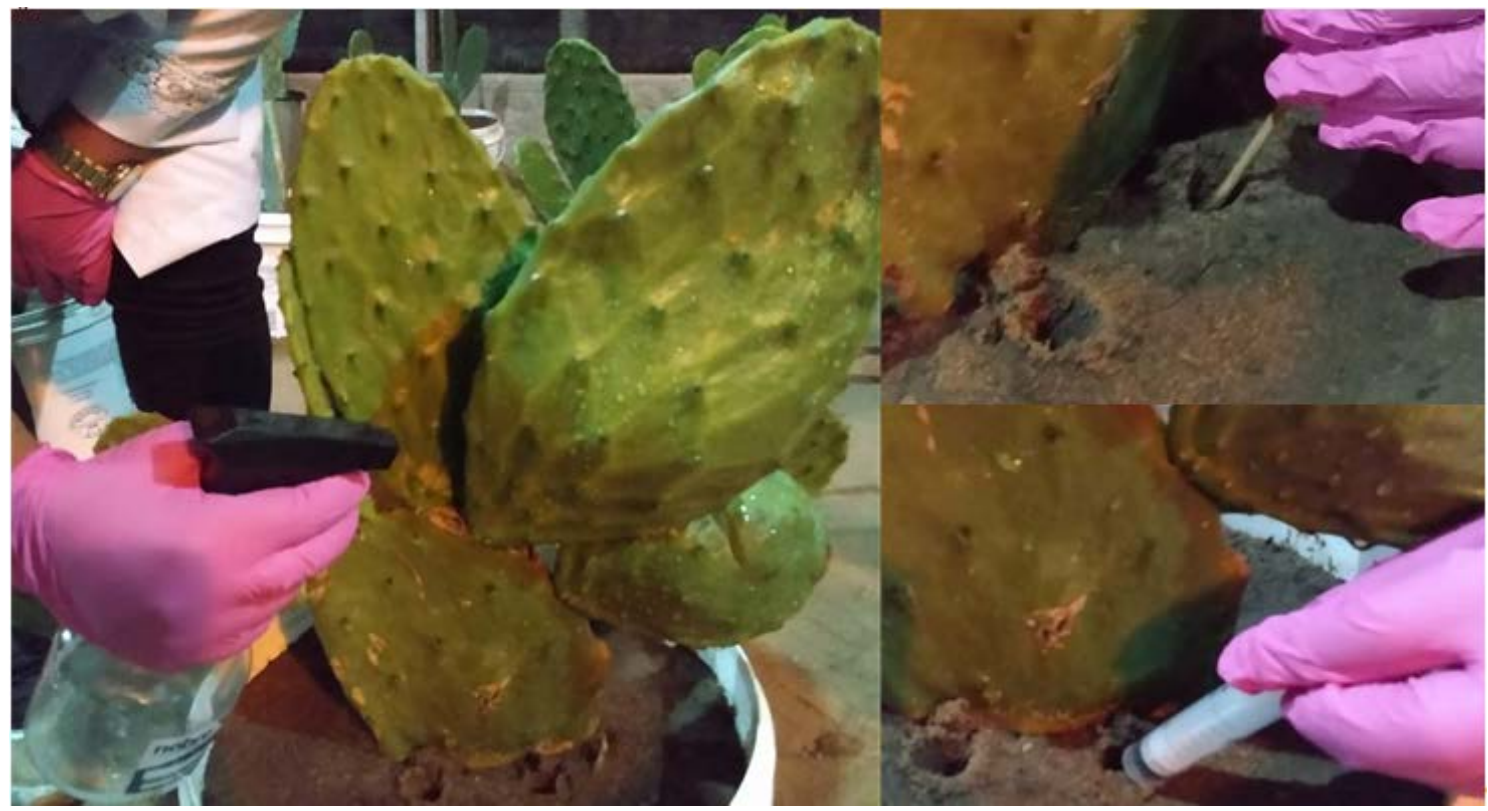

Figura 1. Cladódios de Opuntia spp. sendo pulverizados e aplicação via solo de indutores de resistência bióticos e abióticos.

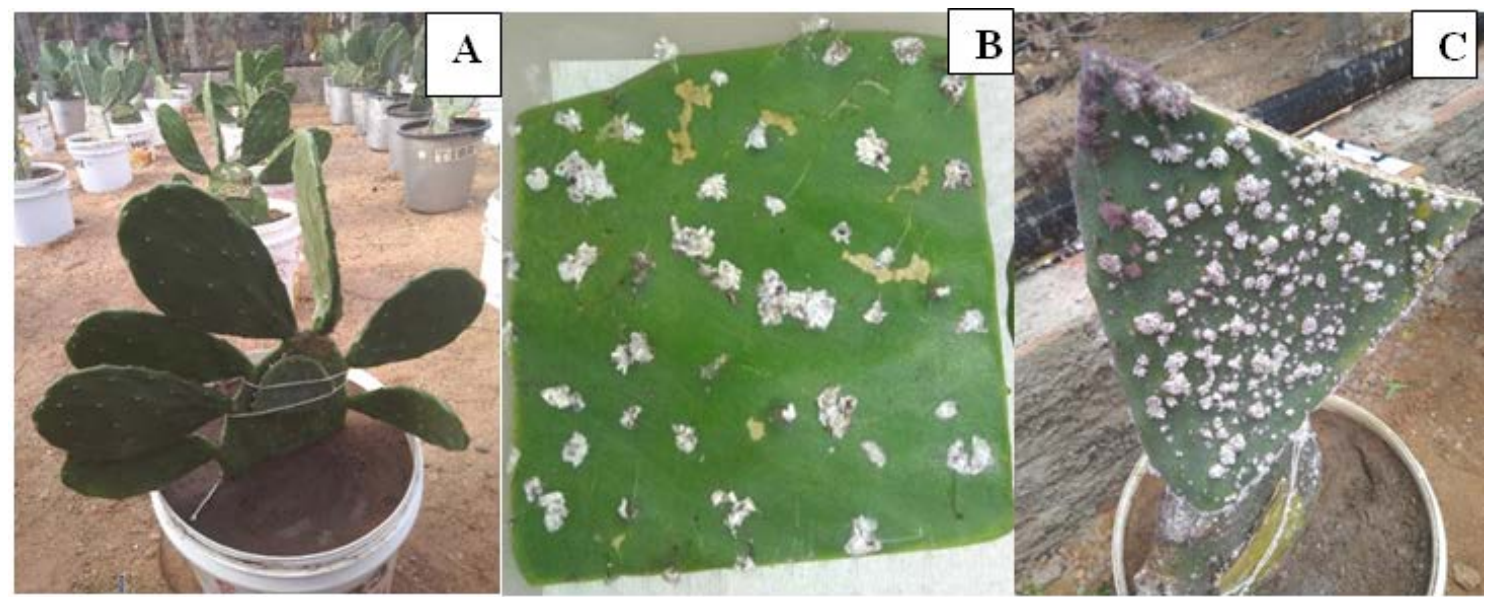

Figura 2. Planta de $O$. atropes infestada artificialmente (A), fragmento de cladódio de palma forrageira com colônias de D. opuntiae (B), planta na fase final do experimento, O. ficus indica (C).

Foi avaliado o número de colônias que se desenvolviam na planta em quatro períodos distintos. A primeira avaliação foi realizada 20 dias após a infestação e as seguintes 40,60, 80 dias, sendo quantificadas por meio de contador de batidas de quatro dígitos (estático analógico) para obtenção do número total por tratamento.

0 delineamento experimental utilizado foi o inteiramente casualizado, com esquema fatorial foi 3x8x4 ( 3 genótipos de palma, 8 indutores de resistência e 4 períodos de contagem). Os dados foram avaliados pela análise de variância (ANOVA), utilizando o programa estatístico SISVAR (versão 5.6) e as médias comparadas pelo teste de Scott-Knott, adotando nível de significância de 5\%. 


\section{Resultados e discussão}

O solo foi classificado como arenoso, apresentando os seguintes teores para argila, areia e silte, respectivamente, 60,0 g. $\mathrm{kg}^{-1}, 920,0$ g.kg-1 e 20,0 g. $\mathrm{kg}^{-1}$. Para matéria orgânica

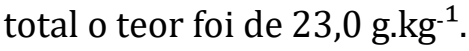

Tabela 1. Análise química do solo utilizado no experimento, coletado a uma profundidade de $0-20 \mathrm{~cm}$.

\begin{tabular}{|c|c|c|c|c|c|c|c|c|c|c|c|c|c|c|c|}
\hline $\mathbf{p H}$ & \multicolumn{10}{|c|}{$\mathbf{m g ~ d m}^{\mathbf{3}}$} & \multicolumn{6}{|c|}{$\mathbf{c m o l}_{\mathbf{c}} \mathbf{d m}^{\mathbf{3}}$} & (V\%) \\
\cline { 2 - 17 } & $\left.\mathbf{~} \mathbf{H}_{\mathbf{2}} \mathbf{0}\right)$ & $\mathbf{N a}$ & $\mathbf{S}$ & $\mathbf{B}$ & $\mathbf{C u}$ & $\mathbf{F e}$ & $\mathbf{M n}$ & $\mathbf{P}$ & $\mathbf{Z n}$ & $\mathbf{C a}$ & $\mathbf{m g}$ & $\mathbf{A l}$ & $\mathbf{H}+\mathbf{A l}$ & $\mathbf{C T C}$ & $\mathbf{S B}$ \\
\hline 6,20 & 78 & 1,0 & 5 & 0,25 & 0,9 & 6,7 & 23 & 36 & 1 & 1,9 & 0,5 & 0,0 & 3,4 & 6,00 & 43 \\
\hline
\end{tabular}

Os três genótipos na primeira avaliação realizada aos 20 dias não apresentaram diferenças estatísticas entres os tratamentos com presença da D. Opuntiae. Quando foi realizada a comparação entre eles, o genótipo susceptível apresentou maiores médias de colônias. Aoyama e Labinas (2012) afirmaram que as interações insetos-plantas envolvem processos ao longo do tempo sendo complexas e dinâmicas, pois tanto um como ou outro são passíveis de mudanças ao longo do cultivo, contudo no início as mudanças são discretas (Tabela 2).

Tabela 2. Quantitativo de colônias de D. opuntiae em três genótipos de palmas do gênero Opuntia, submetidas a indutores de resistência em quatro períodos após infestação artificial.

\begin{tabular}{|c|c|c|c|c|c|c|c|c|}
\hline \multicolumn{9}{|c|}{20 (Dias) } \\
\hline & TRIC 24 & TRIC 23 & AUR & QUIT & AS & BABA & TCC & TSC \\
\hline O. atropes & $51 \mathrm{Ab}^{\mathrm{d}}$ & $54,25 \mathrm{Aa}^{\mathrm{d}}$ & $55,25 \mathrm{Ab}^{\mathrm{d}}$ & $53 \mathrm{Ab}^{\mathrm{d}}$ & $55,50 \mathrm{Aa}^{\mathrm{d}}$ & $53,75 \mathrm{Ab}^{\mathrm{d}}$ & $53,50 \mathrm{Ab}^{\mathrm{d}}$ & $0 \mathrm{Ba}$ \\
\hline O. ficus indica & $108,25 \mathrm{Aa}^{\mathrm{d}}$ & $76,25 \mathrm{Aa}^{\mathrm{c}}$ & $115,50 \mathrm{Aa}^{\mathrm{c}}$ & $107,25 \mathrm{Aa}^{\mathrm{d}}$ & $73,75 \mathrm{Aa}^{\mathrm{d}}$ & $91,50 \mathrm{Aa}^{\mathrm{d}}$ & $101,25 \mathrm{Aa}^{\mathrm{d}}$ & $0 \mathrm{Ba}$ \\
\hline O. stricta & $51 \mathrm{Ab}$ & $50,50 \mathrm{Aa}$ & $52,25 \mathrm{Ab}$ & $51,5 \mathrm{Ab}$ & $52 \mathrm{Aa}$ & $52,75 \mathrm{Ab}$ & $52,50 \mathrm{Ab}$ & $0 \mathrm{Ba}$ \\
\hline \multicolumn{9}{|c|}{40 (Dias) } \\
\hline O. atropes & $94,75 \mathrm{Cb}^{\mathrm{c}}$ & $186,25 \mathrm{Bb}^{\mathrm{c}}$ & $142,75 \mathrm{Bb}^{\mathrm{c}}$ & $116,25 \mathrm{Cb}^{\mathrm{c}}$ & $174,50 \mathrm{Bb}^{\mathrm{c}}$ & $222 \mathrm{Ab}^{\mathrm{c}}$ & $328,25 \mathrm{Ab}^{\mathrm{c}}$ & $0 \mathrm{Da}$ \\
\hline O. ficus indica & $377 \mathrm{Ba}^{\mathrm{c}}$ & $744 \mathrm{Aa}^{\mathrm{b}}$ & $819 \mathrm{Aa}^{\mathrm{b}}$ & $428,75 \mathrm{Ba}^{\mathrm{b}}$ & $277 \mathrm{Ca}^{\mathrm{c}}$ & $637 \mathrm{Aa}^{\mathrm{c}}$ & $807,25 \mathrm{Aa}^{\mathrm{c}}$ & $0 \mathrm{Da}$ \\
\hline O. stricta & $51 \mathrm{Ab}$ & $52,50 \mathrm{Ac}$ & $53,75 \mathrm{Ac}$ & $54,25 \mathrm{Ac}$ & $53,2 \mathrm{Ac}$ & $52,75 \mathrm{Ac}$ & $54,25 \mathrm{Ac}$ & $0 \mathrm{Ba}$ \\
\hline \multicolumn{9}{|c|}{60 (Dias) } \\
\hline O. atropes & $231,50 \mathrm{Ab}^{\mathrm{b}}$ & $310 \mathrm{Ab}^{\mathrm{b}}$ & $285 \mathrm{Bb}^{\mathrm{b}}$ & $226 \mathrm{Bb}^{\mathrm{b}}$ & $274,25 \mathrm{Ba}^{\mathrm{b}}$ & $322,25 \mathrm{Ab}^{\mathrm{b}}$ & $435,50 \mathrm{Ab}^{b}$ & $0 \mathrm{Ca}$ \\
\hline O. ficus indica & $506,50 \mathrm{Ba}^{\mathrm{b}}$ & $895 \mathrm{Aa}^{\mathrm{b}}$ & $1.031,25 \mathrm{Aa}^{\mathrm{a}}$ & $515,25 \mathrm{Ba}^{\mathrm{b}}$ & $425,50 \mathrm{Ba}^{\mathrm{b}}$ & $814 A a^{b}$ & $958 \mathrm{Aa}^{\mathrm{b}}$ & $0 \mathrm{Ca}$ \\
\hline O. stricta & $53,50 \mathrm{Ac}$ & $53,50 \mathrm{Ac}$ & $58 \mathrm{Ac}$ & $58,50 \mathrm{Ac}$ & $55 \mathrm{Ac}$ & $55,50 \mathrm{Ac}$ & $56,50 \mathrm{Ac}$ & $0 \mathrm{Ba}$ \\
\hline \multicolumn{9}{|c|}{80 (Dias) } \\
\hline O. atropes & $378,75 \mathrm{Bb}^{\mathrm{a}}$ & $545,50 \mathrm{Ab}^{\mathrm{a}}$ & $504,25 \mathrm{Ab}^{\mathrm{a}}$ & $404,75 \mathrm{Bb}^{\mathrm{a}}$ & $504,25 \mathrm{Aa}^{\mathrm{a}}$ & $491,50 \mathrm{Bb}^{\mathrm{a}}$ & $602,75 \mathrm{Ab}^{\mathrm{a}}$ & $0 \mathrm{Da}$ \\
\hline O. ficus indica & $706 \mathrm{Ba}^{\mathrm{a}}$ & $1.153 \mathrm{Aa}^{\mathrm{a}}$ & $1.184,5 \mathrm{Aa}^{\mathrm{a}}$ & $655,75 \mathrm{Ba}^{\mathrm{a}}$ & $719 \mathrm{Ba}^{\mathrm{a}}$ & $1.245 .5 \mathrm{Aa}^{\mathrm{a}}$ & $1.424 \mathrm{Aa}^{\mathrm{a}}$ & $0 \mathrm{Ca}$ \\
\hline O. stricta & $56,25 \mathrm{Ac}$ & $55 \mathrm{Ac}$ & $61,25 \mathrm{Ac}$ & $62 \mathrm{Ac}$ & $61,25 \mathrm{Ab}$ & $60 \mathrm{Ac}$ & $60,75 \mathrm{Ac}$ & 0Ba \\
\hline
\end{tabular}

Letras maiúsculas na linha comparam a palma com os diferentes tratamentos dentro da coleta. Letras minúsculas na coluna comparam o tratamento dentro de cada genótipo de palmas entre si na referida coleta. Letras em exponencial comparam o genótipo dentro do tratamento por coletas Letras semelhantes não diferem pelo teste de Scott-Knott a 5\%. T. viride URM 6824 (TRIC 24), T. viride URM 6823 (TRIC 23); Aureobasidium pullulans URM 6874 (AUR); Quitosana (QUIT); Ácido salicílico (AS); Ácido aminobutírico (BABA); Testemunha com cochonilha (TCC); Testemunha sem cochonilha (TSC). 
Quando as plantas foram avaliadas pela segunda vez, a variedade $O$. ficus indica as plantas submetidas aos tratamentos dos indutores Ácido salicílico, T. viride URM6824 e o quitosana apresentaram menores quantitativos médios de colônias (277, 377 e 428,7), respectivamente, sendo estatisticamente iguais, neste período as testemunhas positivas já tinham um número médio de colônias de 807,25. Para variedade 0 . atropes (tolerante) os tratamentos com $T$. viride URM6824 e quitosana foram os que apresentaram menores quantitativos, já na 0 . stricta (resistente) os tratamentos com os indutores não diferiram das testemunhas. Vasconcelos et al. (2009) realizando experimento as variedades $O$. stricta e 0 . atropes infestadas artificialmente com 10 colônias por plantas, aos 47 dias após IA as plantas resistentes tinham de 10-12 e as tolerantes 81-120 colônias.

Quando a análise estatística foi entre os genótipos dentro no mesmo período de avaliação eles foram estatisticamente diferentes. Lopes et al. (2018) avaliaaram o fungo entomopatogênico Isaria farinosa ESALQ1355 frente 0 . ficus indica e concluíram o fungo nas condições testadas não foi patogênica sobre as fêmeas adultas de $D$. opuntiae, causando a morte de $8 \%$.

Na terceira avaliação já aos 60 dias após I.A no genótipo 0 . ficus indica nas plantas submetido aos tratamentos: T. viride URM6824, quitosana e ao Ácido salicílico mantiveram-se os menores quantitativos de colônias (506,50, 425,50 e 425,50) enquanto nas plantas que foram tratadas com $A$. pullulans URM 6874 apresentaram média de 1.031,25 colônias. Carvalho et al. (2007) avaliaram a atividade parasítica sobre a cochonilha dos fungos: Beauveria, Metarrhizium, Paecillomyces e Trichoderma, concluíram que tais fungos não exerceram nenhuma atividade parasítica sobre as cochonilhas que infestavam os cladódios testados. Bezerra (2018) avaliou períodos de infestações com o genótipo 0 . ficus indica, teve como resultado que aos 60 dias as plantas possuíam 3.936,44 colônias e tais plantas apresentaram uma menor quantidade de matéria seca e lignina em comparação com as plantas no início do experimento.

Em experimento realizado na Estação Experimental de Lagoa Seca-PB, os resultados demonstraram o poder elevado de dispersão em condição de campo, uma planta inicialmente com dez colônias, após 60 dias foi observado o valor médio de 171 colônias por planta, fato que preconiza que o nível de controle da cochonilha do carmim é menos de 10 colônias por planta (Aguiar et al., 2019).

Falcão et al. (2013) averiguaram o perfil metabólico mediante a pressão de infestação à planta, obtiveram como resultados que as plantas de IPA-100003 com 100\% de infestação apresentaram uma queda de proteína solúvel, nos genótipos resistente (IPA-200016) e o tolerante (IPA 200008) o resultado foi o oposto, tiveram elevado teor de proteína e aminoácidos, tal comportamento pode estar relacionado a utilização destes metabólitos no metabolismo secundário, os quais interferem nas características do sabor tornando-o menos atraente, com a maior mobilização de mecanismos de defesas resulta em um maior gasto energético e menor acúmulo de biomassa.

No presente estudo quando foi realizada a avaliação aos 80 dias, no genótipo susceptível (IPA-100003) a testemunha positiva apresentava uma média de 1424 colônias distribuídas por toda a planta, alguns cladódios já estavam totalmente tomados pelas colônias, formando uma espécie de tapete. No genótipo tolerante (IPA200008) a testemunha positiva mesmo não diferindo estatisticamente entre os tratamentos: $T$. viride URM 6823, A. pullulans URM 6874, quitosana; ácido salicílico teve uma média de 602,75 colônias. No genótipo resistente a média da testemunha positiva foi de 60,75 colônias também não diferiu entre os tratamentos, as colônias presentes nos cladódios desenvolveram-se embaixo do fragmento que foi usado como veículo na infestação e não houve fixação nos demais cladódios. 


\section{Conclusões}

Os indutores T. viride URM 6824, quitosana e o ácido salicílico proporcionam menores quantitativos de colônias nas do genótipo $O$. ficus indica. Já no genótipo $O$. atropes os que promovem menores valores é T. viride URM 6824, quitosana e o BABA.

\section{Agradecimentos}

A Fundação de Amparo à Ciência e Tecnologia do estado de Pernambuco (FACEPE) pelo apoio e concessão da bolsa referente ao projeto de pesquisa (IBPG-0729-5.01/18).

\section{Conflito de interesses}

Os autores declaram não haver conflito de interesses.

\section{Referências}

Aoyama, E. M.; Labinas, A. M. Características estruturais das plantas contra a herbivoria por insetos. Enciclopédia Biosfera, Centro Científico Conhecer, v. 8, p. 366-386, 2012.

Batista, V. C. V.; Pereira, I. M. C.; Paula-Marinho, S. O.; Canuto, K. M.; Pereira, R. C. A.; Rodrigues, T. H. S.; Daloso, D. M.; Gomes-Filho, E.; Carvalho, H. H. Salicylic acid modulates primary and volatile metabolites to alleviate salt stress-induced photosynthesis impairment on medicinal plant Egletes viscosa. Environmental and Experimental Botany, v. 167, 103870, 2019. https://doi.org/10.1016/j.envexpbot.2019.103870

Bezerra, J. D. C. Mecanismos de resistência em genótipos de palma forrageira à infestação por Dactylopius opuntiae associados ao perfil metabólico. Areia: Universidade Federal da Paraíba, 2018. (Tese de doutorado).

Bozoudi, D.; Tsaltas, D. The multiple and versatile roles of Aureobasidium pullulans in the vitivinicultural sector. Journal Fermentation, v. 4, n. 4, 85, 2018. https://doi.org/ $10.3390 /$ fermentation 4040085

Canuto, C.; Andrade, D. S.; Lima, E. M.; Goes, M. C. C.; Almeida, M. M. V.; Lima, J. R. S. Biochar e esterco bovino aumentam a eficiência no uso de água da alface. Diversitas Journal, v. 4, n. 3, p. 1082-1091, 2019. https://doi.org/10.17648/diversitas-journal-v4i3.822

Carvalho, R. A.; Lopes, E. B.; Silva, A. C.; Leandro, R. S.; Campos, V. B. Controle alternativo da cochonilha-do-carmim em palma forrageira no cariri paraibano. Brasília: MMA, 2007.

Clima-Date.Org. Garanhuns clima (Brasil). 2020. Disponível em: <https://pt.climatedata.org/a,merica-do-sul/brasil/pernambuco/garanhuns-4458/>. Acesso em: 25 ago. 2020.

Delgado-Oramas, B. P. La resistencia inducida como alternativa para el manejo de plagas en las plantas de cultivo. Revista de Protección Vegetal, v. 35, p. 1-12, 2020.

Falcão, H. M.; Oliveira, M. T.; Mergulhão, A. C.; Silva, M. V.; Santos, M. G. Ecophysiological performance of three Opuntia ficus-indica cultivars exposed to carmine cochineal under field conditions. Scientia Horticulturae, v. 150, p. 419-424. 2013. https://doi.org/ 10.1016/j.scienta.2012.11.021

Ferraz, L. P.; Cunha, T.; Silva, A. C.; Kupper, K. C. Biocontrol ability and putative mode of action of yeasts against Geotrichum citri-aurantii in citrus fruit. Microbiological Research, v. 188/189, p. 72-79, 2016. https://doi.org/10.1016/j.micres.2016.04.012 
Gorri, J. E. R.; Ribeiro, L.; Reais, D. S.; Morais, K. D. B.; Costa, N. C. R. Silva, E. M. A Química na produção vegetal. Rio Paranaíba: Dos Autores, 2017.

INMET - Instituto Nacional de Meteorologia. 2020. Disponível em: $<$ https://portal.inmet.gov.br>. Acesso em: 25 ago. 2020.

Lira, M. A. Palma forrageira: cultivo e usos. Cadernos do Semiárido: Riqueza \& Oportunidades, v.7, p.1-58, 2017. Disponível em: <http://www.ipa.br/novo/pdf/ cadernos-do-semiarido/7---palma-forrageira---cultivo-e-usos.pdf>. Acesso em: 23 ago. 2020.

Lopes, R. S.; Oliveira, L. G.; Lima, G.; Costa, A. F.; Lima, E. A. L. A.; Lima, V. L. M. Controle biológico e alternativo de Dactylopius opuntiae por fungo entomopatogênico e extratos vegetais em plantação de Opuntia ficus-indica (Pernambuco/Brasil). Pesquisa Agropecuária Pernambucana, v. 23, p. 1-4, 2018. https://doi.org/10.12661/ pap.2018.007

Marques, O. F. C.; Gomes, L. S. P.; Mourthé, M. H. F.; Braz, T. G. S.; Pires Neto, O. S. Palma forrageira: cultivo e utilização na alimentação de bovinos. Caderno de Ciências Agrárias, v. 9, n. 1, p. 75-93, 2017.

Melo, L. G. L.; Candido e Silva, E. K.; Campos Neto, J. R. M.; Lins, S. R. O.; Rodrigues, A. A. C.; Oliveira, S. M. A. Indutores de resistência abióticos no controle da fusariose do abacaxi. Pesquisa Agropecuária Brasileira, v. 51, p. 1703-1709, 2016. https://doi.org/10.1590/ s0100-204x2016001000001

Palafox-Luna, J. A.; Rodríguez-Leyva, E.; Lomeli-Flores, J. R.; Vigueras-Guzmán, A. L.; Vanegas-Rico, J. M. Ciclo de vida y fecundidad de Dactylopius opuntiae (Hemiptera: Dactylopiidae) en Opuntia ficus-indica (Caryophyllales: Cactaceae). Agrociencia, v. 52, n. 1, 2018.

Pascholatti, S. F.; Souza, V. H. M.; Cardoso Filho, J. A. Indução de resistência por Trichoderma. In: Meyer, M. C. Trichoderma: uso na agricultura. Brasília: EMBRAPA, 2019. p. 235-254.

Pfliegler, W. P.; Pusztahelyi, T.; Pócsi, I. Micotoxinas: Prevention and descontamination by yeasts. Journal of Basic Microbiology, $\quad$ v. 55, n. 2, p. 805-818, 2015. https://doi.org/10.1002/jobm.201400833

Silva, É. S.; Gomes, I. A.; Melo, L. J.; Medeiros, W. P.; Medeiros, M. B. Características da palma forrageira Opuntia spp. com potencial à resistência à cochonilha do carmim Dactylopius opuntiae (Cockerell) (Hemiptera: Dactylopiidae). Revista Brasileira de Gestão Ambiental e Sustentabilidade, v. 7, n. 17, p.1533-1541, 2020. https://doi.org/10.21438/rbgas(2020)071731

Teixeira, P. C.; Donagemma, G. K.; Fontana, A.; Teixeira, W. G. (Eds.). Manual de métodos de análise de Solo. 3. ed. rev. e ampl. Brasília: EMBRAPA, 2017. Disponível em: $<$ https://ainfo.cnptia.embrapa.br/digital/bitstream/item/181717/1/Manual-deMetodos-de-Analise-de-Solo-2017.pdf>. Acesso em: 25 ago. 2020.

Vasconcelos, A. J. V.; Lira, M. A.; Cavalcanti, V. L. B.; Santos, M. V. F.; Willadino, L. Seleção de clones de palma forrageira resistentes à cochonilha-do-carmim (Dactylopius sp). Revista Brasileira de Zootecnia, v. 38, p. 827-831, 2009. https://doi.org/10.1590/S151635982009000500007 
Wang, W.; Zhou, P.; Mo, X.; Hu, L.; Jin, N.; Chen, X.; Yu, Z.; Meng, J.; Erb, M.; Shang, Z.; Gatehouse, A. M. R.; Wu, J.; Lou, Y. Induction of defense in cereals by 4-fluorophenoxyacetic acid suppresses insect pest populations and increases crop yields in the field. Proceedings of the National Academy os Sciences of the United States of America, v. 117, p. 12017-12028, 2020. https://doi.org/10.1073/pnas.2003742117 seja devidamente citada. 